\title{
Current clinical status and vascular complications among patients with type 2 diabetes mellitus at tertiary hospitals in Malaysia
}

\begin{abstract}
Objective: To assess the prevalence of diabetic vascular complications and cardiovascular risk factors control in type 2 diabetic patients at tertiary settings. Methods: This crosssectional study was conducted among 313 patients diagnosed with type 2 diabetes mellitus (T2DM) at two tertiary referral hospitals in Malaysia. Data regarding socio-demographics, macro- and microvascular complications, family health history, blood pressure, anthropometric indices, glycaemic control, and lipid profile were obtained from medical records, face-to-face interview and physical examination. Results: The mean age of patients was $55.7 \pm 9.2$ years, mean diabetes duration was $10.1 \pm 8.1$ years, and $52.1 \%$ were females. Approximately $36.1 \%$ patients had cardiovascular disease (CVD). There were high prevalence of established coronary artery disease $(30.7 \%)$, cerebrovascular disease $(10.2 \%)$, and peripheral vascular disease $(5.1 \%)$. Peripheral neuropathy, diabetic nephropathy and retinopathy were present in $41.5 \%, 17.6 \%$ and $15.0 \%$ patients respectively. Only $14.1 \%$ of the patients reached optimal HbAlc level and $21.1 \%$ patients achieved target fasting plasma glucose. The overall prevalence of dyslipidemia was $89.1 \%$, hypertension was $80.2 \%$, and obesity was $35.9 \%$ (BMI) and $86.5 \%$ (waist-to-hip ratio). Conclusions: Diabetic vascular complications were highly prevalent among the type 2 diabetic patients. Cardiovascular risk factors control was suboptimal. Both awareness and application of recommended guidelines need to be reinforced.
\end{abstract}

Keyword: Diabetic vascular complications; Cardiovascular disease; Neuropathy;

Nephropathy; Retinopathy; Cardiovascular risk factors; Type 2 diabetes mellitus;

Tertiary referral hospitals 